\title{
Adjuvant chemotherapy for older women with breast cancer
}

\author{
H. B. Muss \\ Hematology/Oncology Unit, University of Vermont and Vermont Cancer Center, Burlington, VT, USA
}

\begin{abstract}
Many older women today with early-stage breast cancer have estimated survivals exceeding 5-10 years at the time of diagnosis and should be considered for systemic adjuvant chemotherapy. When available and if eligible, such patients should be offered clinical trials participation. Given the median age for onset of breast cancer, trials should also be developed to specifically investigate the disease in this age group. Oncologists caring for elders need to educate their colleagues as well as their patients concerning the pitfalls of age bias and the potential hazard of under-treatment and poorer outcomes.
\end{abstract}

Keywords: Adjuvant therapy; Chemotherapy; Elderly; Primary breast cancer

\section{Introduction}

There will be 1437000 new cancer cases and 566000 cancer deaths in the United States in 2008 [1]. The incidence of cancer in the US dramatically increases with age with a median age at diagnosis of 67 years and a median age at death of 73 years. Breast cancer is the most common cancer in women, and in the US is second only to lung cancer as the leading cause of cancer death. About 182000 new cases of invasive breast cancer and 41000 breast cancer deaths are estimated for 2008 [1]. The median age at diagnosis of breast cancer in the US is 61 years and median age at death is 69 years. The median age of incidence of breast cancer is similar to other affluent nations, making breast cancer a major cause of cancer morbidity and mortality for much of the world's older women. Although older breast cancer patients have slightly

Correspondence to: H. B. Muss, MD, Professor of Medicine, Hematology/ Oncology Unit, University of Vermont and Vermont Cancer Center, Burlington, VT 05405, USA. E-mail: hyman.muss@uvm.edu

Received: 10/07/08

Accepted: 21/07/08

$\mathrm{BCO} / 645 / 2008 / \mathrm{FO}$ better tumor characteristics than younger women (they are more likely to be hormone receptorpositive, have lower-grade tumors, node-negative, and HER-2-negative tumors) [2], survival is similar or possibly even worse than for younger patients after adjusting for stage and receptor status $[3,4]$.

Major advances have been made in the adjuvant systemic therapy of breast cancer, with both endocrine therapy and chemotherapy playing a major role in improving survival [5]. Nevertheless, older patients continue to be under-represented in adjuvant breast cancer trials $[6,7]$, especially in randomized trials involving chemotherapy [5]. Increasing evidence suggests that healthy older patients get similar benefits as younger patients with newer stateof-the-art chemotherapy treatments $[8,9]$ although with greater treatment-related morbidity and mortality $[9,10]$. Anthracycline therapy specifically has been associated with increased risks of congestive heart failure [11] and leukemia [12]. This review focuses on adjuvant chemotherapy.

\section{Age, comorbidity, and life expectancy}

Older patients are less likely to be considered for adjuvant chemotherapy based on age bias alone 
but older women in good health can have a considerable life expectancy. For example, a healthy 65 -year-old woman has an average remaining life expectancy of 20 years, a healthy 75-year old of 13 years, and a healthy 85-year old of 7 years [13]. These data indicate that adjuvant chemotherapy should be considered for many healthy older women with a breast cancer at high risk of recurrence over a 10-year period. Factoring in how other comorbid illness competes with breast cancer in lowering life expectancy can be estimated using computer models such as Adjuvant! [14] (see also www.adjuvantoline.com). Estimates of the severity of comorbidity can be added to the model and greatly help in decision making.

\section{Selecting patients for treatment}

Historically, patients have been selected for treatment based on clinical trial designs that categorized patients by nodal status (node-negative or nodepositive) and hormone receptor status (positive or negative). New insights into the biology of breast cancer [15] have suggested that patients might be better selected by dividing them into three specific groups: (1) those with hormone receptor-positive, HER-2-negative tumors (by far the largest group and comprising the majority of older women), (2) those with HER-2-positive tumors irrespective of hormone receptor status (about 10-15\% of older women), and (3) those with hormone receptor-negative, HER2-negative tumors - 'triple-negatives' (about 15\% of older patients). Such a division allows one to apply data from more recent trials in making the best treatment decision.

For the group with hormone receptor-positive, HER-2-negative tumors, adjuvant therapy with either tamoxifen or aromatase inhibitors is the adjuvant therapy of choice, and the major decision concerning the role of chemotherapy is calculating its potential added value to endocrine therapy. For elders with node-negative, estrogen receptor (ER)positive tumors who are candidates for 5 years of tamoxifen, the recently developed OncotypeDx assay $[16,17]$ can estimate the added value of chemotherapy and can be of great help in decision making; few older patients are likely to benefit from chemotherapy in this setting but a small percentage might derive major benefit [9]. The TAILORx trial (ECOG-PACCT-1; http://www.cancer.gov) in the US and the MINDACT trial (EORTC 10041 (BIG 3-04; http://www.eortc.be) in Europe use tumor genetic assessments to randomize patients at moderate risk of recurrence to chemotherapy or not; older patients eligible for these trials should be offered participation. A flaw in the MINDACT trial is an age restriction of 18-70 years. For women in this group with positive lymph nodes, the decision is more complicated; use of Adjuvant! can help with these decisions, and factoring in comorbidity is an essential element assessing the value of chemotherapy on improving survival.

Older women and younger women with HER2-positive tumors have higher risks of early relapse and should be considered for trastuzumab therapy in addition to chemotherapy [18,19]. For older women treated with trastuzumab, cardiac toxicity is a major concern as age is a risk factor for an increased risk of cardiac toxicity and CHF $[20,21]$. Older patients need to be carefully monitored, and for some with a history of hypertension or heart disease, initiation of ACE-inhibitors and betablockers might be considered prior to starting trastuzumab to minimize the cardiac risks. Also, elders should be considered for non-anthracyclinecontaining regimens with trastuzumab such as docetaxel and carboplatin [22] or docetaxel and cyclophosphamide [23] to minimize cardiac risk. Not all older patients with HER-2-positive tumors require trastuzumab. For those with small, nodenegative tumors a centimeter or less, the added value of trastuzumab is small and risks may outweigh benefits.

Older women with triple-negative breast cancer and estimated non-breast cancer estimated survival greater than 5 years should be offered state-ofthe-art chemotherapy including taxane-containing regimens $[24,25]$. This aggressive strategy is supported by a recent analysis of the Early Breast Cancer Trialists Collaborative Group (EBCTCG) comparing chemotherapy or not in women with ER-poor tumors, which showed a 10-year reduction in breast cancer mortality of $8 \%$ in women younger than 50 years, and $6 \%$ in women aged $50-69$ years [26]. Of note, these impressive survival benefits were achieved with older, non-taxane-containing regimens, with almost half of these patients receiving cyclophosphamide, methotrexate, and fluorouracil. In an analysis of data from the Surveillance, Epidemiology and End Results Program (SEER), older women with node-positive breast cancer and those with higher risk node-negative breast cancer derived significant survival benefits from chemotherapy [27]. A first analysis of CALGB trial 49907, which compared standard chemotherapy (either CMF or doxorubicin and cyclophosphamide (AC)) with capecitabine in women aged 65 and older with early-stage breast cancer, showed that standard chemotherapy was superior to capecitabine, especially in those with receptornegative tumors [28]. Although the HER-2 status of the patients from the CALGB analysis [24], the 
Table 1. Clinical trials specifically designed for older patients with breast cancer.

\begin{tabular}{llll}
\hline Trial & Treatment & N (actual) & Status \\
\hline CALGB 49907 & AC/CMF vs. capecitabine & $600(600+)$ & Closed \\
ICE (BIG) & Ibandronate \pm capecitabine & $1400(1350)$ & $\mathrm{N} \pm$, ER+ and AI \\
CASA (BIG) & Caelyx vs. none Caelyx vs. po CM & Closed after 42 pts & ER- and PR- \\
ELDA (NCI-Naples) & Docetaxel vs. CMF & 300 (just started) & mod-high risk \\
ACTION (UK) & AC or EC vs. none & 1000 (just started) & ER- \\
\hline
\end{tabular}

meta-analysis of the EBCTCG [26], and the SEER database [27] was not available, it is likely that about $80 \%$ or more of these patients had triplenegative breast cancer and one would expect even better results if trastuzumab were given to the HER2-positive patients in these trials.

\section{Clinical trials}

Older patients continue to be under-represented in clinical trials and clinicians should be encouraged to offer trial participation to older patients. Available data suggest that when offered trials, older and younger patients have similar rates of participation, approximating 50\% [29]. Another strategy to improve accrual is to design trials specifically for older patients, and current trials for older women with early breast cancer are presented in Table 1. In addition, newer trials with more aggressive regimens would benefit by incorporating a CGA assessment tool for older patients as part of the trial. The shortage of trained geriatricians has lead to the development of shorter instruments that can accurately predict functional decline and mortality risk [30-32]. Helping to identify which patients are at greatest risk for treatment toxicity and loss of function prior to treatment would be of great value to patients and physicians. A gap in knowledge related to management of frail patients with early breast cancer [33]. Specific trials for this group of patients are needed.

\section{Conclusions}

Many older women today with early-stage breast cancer have estimated survivals exceeding 5-10 years at the time of diagnosis and should be considered for systemic adjuvant chemotherapy. When available and if eligible, such patients should be offered clinical trials participation. Outside of a trial, management guidelines for older women with breast cancer have been recently developed by the international Society of Geriatric Oncology and provide excellent guidance for patient management [34]. In addition, several excellent recent reviews of this topic are available [35,36]. Oncologists caring for elders need to educate their colleagues as well as their patients concerning the pitfalls of age bias and the potential hazard of under-treatment and poorer outcomes.

\section{References}

1. Jemal A, Siegel R, Ward E, et al. Cancer statistics, 2008. CA Cancer J Clin 2008; 58: 71-96.

2. Diab SG, Elledge RM, Clark GM. Tumor characteristics and clinical outcome of elderly women with breast cancer. J Natl Cancer Inst 2000; 92: 550-556.

3. Holli K, Isola J. Effect of age on the survival of breast cancer patients. Eur J Cancer 1997; 33: 425-428.

4. Jatoi I, Chen BE, Anderson WF, Rosenberg PS. Breast cancer mortality trends in the United States according to estrogen receptor status and age at diagnosis. $J$ Clin Oncol 2007; 25: 1683-1690.

5. Effects of chemotherapy and hormonal therapy for early breast cancer on recurrence and 15-year survival: an overview of the randomised trials. Lancet 2005; 365: 1687-1717.

6. Sateren WB, Trimble EL, Abrams J, et al. How sociodemographics, presence of oncology specialists, and hospital cancer programs affect accrual to cancer treatment trials. J Clin Oncol 2002; 20: 2109-2117.

7. Hutchins LF, Unger JM, Crowley JJ, Coltman CA Jr, Albain KS. Underrepresentation of patients 65 years of age or older in cancer-treatment trials. $N$ Engl $J$ Med 1999; 341: 2061-2067.

8. Muss HB, Woolf S, Berry D, et al. Adjuvant chemotherapy in older and younger women with lymph nodepositive breast cancer. JAMA 2005; 293: 1073-1081.

9. Giordano SH, Duan Z, Kuo YF, Hortobagyi GN, Goodwin JS. Use and outcomes of adjuvant chemotherapy in older women with breast cancer. J Clin Oncol 2006; 24: 2750-2756.

10. Muss HB, Berry DA, Cirrincione C, et al. Toxicity of older and younger patients treated with adjuvant chemotherapy for node-positive breast cancer: the Cancer and Leukemia Group B Experience. J Clin Oncol 2007; 25: 3699-3704.

11. Pinder MC, Duan Z, Goodwin JS, Hortobagyi GN, Giordano $\mathrm{SH}$. Congestive heart failure in older women treated with adjuvant anthracycline chemotherapy for breast cancer. J Clin Oncol 2007; 25: 3808-3815.

12. Patt DA, Duan Z, Fang S, Hortobagyi GN, Giordano SH. Acute myeloid leukemia after adjuvant breast cancer therapy in older women: understanding risk. $J$ Clin Oncol 2007; 25: 3871-3876. 
13. USLife Expectancy. United States Life Tables, 2003. Available at http://www.cdc.gov/nchs/data/statab/lewk3_ 2003.pdf

14. Ravdin PM, Siminoff LA, Davis GJ, et al. Computer program to assist in making decisions about adjuvant therapy for women with early breast cancer. J Clin Oncol 2001; 19: 980-991.

15. Perou CM, Sorlie T, Eisen MB, et al. Molecular portraits of human breast tumours. Nature 2000; 406: 747-752.

16. Paik S, Shak S, Tang G, et al. A multigene assay to predict recurrence of tamoxifen-treated, node-negative breast cancer. N Engl J Med 2004; 351: 2817-2826.

17. Paik S, Tang G, Shak S, et al. Gene expression and benefit of chemotherapy in women with node-negative, estrogen receptor-positive breast cancer. $J$ Clin Oncol 2006; 24: 3726-3734.

18. Romond EH, Perez EA, Bryant J, et al. Trastuzumab plus adjuvant chemotherapy for operable HER2-positive breast cancer. N Engl J Med 2005; 353: 1673-1684.

19. Piccart-Gebhart MJ, Procter M, Leyland-Jones B, et al. Trastuzumab after adjuvant chemotherapy in HER2positive breast cancer. N Engl J Med 2005; 353: 1659-1672.

20. Perez EA, Suman VJ, Davidson NE, et al. Cardiac safety analysis of doxorubicin and cyclophosphamide followed by paclitaxel with or without trastuzumab in the North Central Cancer Treatment Group N9831 adjuvant breast cancer trial. J Clin Oncol 2008; 26: 1231-1238.

21. Telli ML, Hunt SA, Carlson RW, Guardino AE. Trastuzumabrelated cardiotoxicity: calling into question the concept of reversibility. J Clin Oncol 2007; 25: 3525-3533.

22. Slamon D, Eiermann W, Robert N, et al. Second interim analysis phase III randomized trial comparing doxorubicin and cyclophosphamide followed by docetaxel with doxorubicin and cyclophosphamide followed by docetaxel and trastuzumab with docetaxel, carboplatin and trastuzumab in Her2neu postiive early breast cancer patients [Abstract]. Breast Cancer Res Treat 2006.

23. Jones SE, Savin MA, Holmes FA, et al. Phase III trial comparing doxorubicin plus cyclophosphamide with docetaxel plus cyclophosphamide as adjuvant therapy for operable breast cancer. J Clin Oncol 2006; 24: 5381-5387.

24. Berry DA, Cirrincione C, Henderson IC, et al. Estrogenreceptor status and outcomes of modern chemotherapy for patients with node-positive breast cancer. JAMA 2006; 295: 1658-1667.

25. Hayes DF, Thor AD, Dressler LG, et al. HER2 and response to paclitaxel in node-positive breast cancer. N Engl J Med 2007; 357: 1496-1506.

26. Early Breast Cancer Trialists' Collaborative Group, Clarke M, Coates AS, Darby SC, et al. Adjuvant chemotherapy in oestrogen-receptor-poor breast cancer: patient-level meta-analysis of randomised trials. Lancet 2008; 371: 29-40.

27. Elkin EB, Hurria A, Mitra N, Schrag D, Panageas KS. Adjuvant chemotherapy and survival in older women with hormone receptor-negative breast cancer: assessing outcome in a population-based, observational cohort. J Clin Oncol 2006; 24: 2757-2764.

28. Muss HB, Berry DL, Cirrincione C, et al. and North American Breast Cancer Intergroup. Standard chemotherapy (CMF or AC) versus capecitabine in early-stage breast cancer $(\mathrm{BC})$ patients aged 65 and older: results of CALGB/CTSU 49907 [Abstract]. J Clin Oncol 2008; 26: 8s.

29. Kemeny MM, Peterson BL, Kornblith $A B$, et al. Barriers to clinical trial participation by older women with breast cancer. J Clin Oncol 2003; 21: 2268-2275.

30. Saliba D, Elliott M, Rubenstein LZ, et al. The Vulnerable Elders Survey: a tool for identifying vulnerable older people in the community. J Am Geriatr Soc 2001; 49: 1691-1699.

31. Hurria A, Gupta S, Zauderer M, et al. Developing a cancer-specific geriatric assessment: a feasibility study. Cancer 2005; 104: 1998-2005.

32. Rodin MB, Mohile SG. A practical approach to geriatric assessment in oncology. J Clin Oncol 2007; 25: 1936-1944.

33. Dittus K, Muss HB. Management of the frail elderly with breast cancer. Oncology (Williston Park) 2007; 21: 1727-1734.

34. Wildiers H, Kunkler I, Biganzoli L, et al. Management of breast cancer in elderly individuals: recommendations of the International Society of Geriatric Oncology. Lancet Oncol 2007; 8: 1101-1115.

35. Crivellari D, Aapro M, Leonard R, et al. Breast cancer in the elderly. J Clin Oncol 2007; 25: 1882-1890.

36. Biganzoli L, Aapro M, Balducci L, Crivellari D, Minisini A, Piccart M. Adjuvant therapy in elderly patients with breast cancer. Clin Breast Cancer 2004; 5: 188-195. 